\title{
Under Water Animals Detecting Robot Based on Watershed Algorithm
}

\author{
Le Wang a , Senwei Wang ${ }^{b}$, and Yansong Deng ${ }^{c, *}$ \\ School of Southwest Minzu University, Sichuan 610225, China \\ asxwangll@163.com, b991674069@qq.com, c342547288@qq.com \\ ${ }^{*}$ Corresponding author: Yansong Deng
}

Keywords: Watershed algorithm, OpenCV, Raspberry Pi, The robot.

\begin{abstract}
The underwater animals detecting robot collects images by using a camera, and the watershed algorithm can implement image segmentation. The robot can discern creature under the water with its' advantages of reducing workload and improving work efficiency. The hardware platform of under water animals detecting robot's consists of a Raspberry Pi $2 \mathrm{~B}$ as well as a micro controller. Micro controller controls steering gear to make the robot move freely in the water. OpenCV is an open source computer vision library and Raspberry Pi uses it to image segmentation on the basis of watershed algorithm under Linux system. Watershed algorithm is an image segmentation method based on topology theory, and was put forward to solve the problem of excessive segmentation. According to the results of the experiment, the practicability of the under water animals detecting robot has been proved. Eventually, the robot get an expecting result.
\end{abstract}

\section{Introduction}

With the rapidly development of modern society, the industrial production has brought a great disaster .Such as heavy fog and global warming disaster are sending us a dangerous signal that our home is about to destroying by human. But the earth is only about $29 \%$ of land area, the rest is sea. In the ocean there are many creatures. However, the damage of the marine environment is constantly. The environmental problem has become the most dangerous problem to solve as soon as possible, how to improve the marine environment and protect the marine biological species diversity is every country, everyone should be thinking about. Monitoring the growth condition of marine organisms has become a emergency priority, Traditional artificial diving test has its limits, that is to say, is not only a diver in the face of great personal risk and often difficult to close to the sea creatures .Traditional can only implement long distant observation. So we developed a system. In this paper, the underwater robot can realize autonomous navigation. Its carrying cameras can real-time transmit the image, and the images can be captured. Then login embedded platform through the virtual desktop, and use OpenCV image segmentation to test results under Linux. Provide the reference for researchers to study.

\section{Watershed Algorithm}

\subsection{Watershed Algorithm Principle.}

Watershed algorithm is a kind of image segmentation method based on the theory of the topology. According to the literal meaning, the image is regarded as the terrain. Gray levels of pixels can be regarded as the elevation height, where high grey value is High Mountain and where low grey value is the valley. Some areas are local gray minimum, surrounded by hydrops, known as the reception basin, and the boundary of the basin will constitute the watershed. Put the model into the water, the water flow from the lower part of the terrain, expand slowly every reception basin area. With the injection of water became more and more, water will flow over the low mountain, and flow to the other reception basin area. So two reception basin will be connected, and then form into a region. Two sets the boundary of the basin is dam, adopting some method make the different areas around the dam were not connected by immersion, this is what image segmentation have to complete the task. 


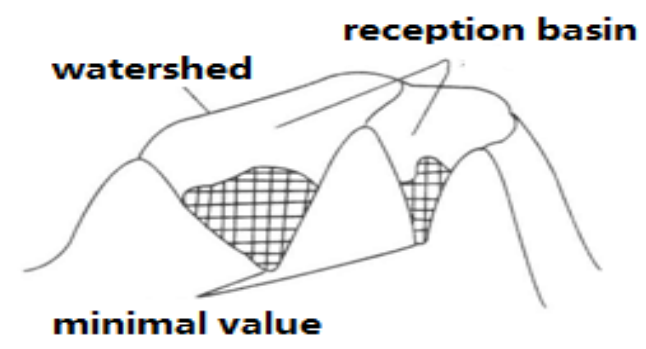

\subsection{Method of Implementation.}

Fig. 1 schematic watershed algorithm

Vincent's watershed algorithm is classic. Vincent algorithm is divided into two steps. First, rank each pixel's gray value from low to high, and then drown the pixels from low to high. For each local minimum value, the height $h$ order domain of influence judgment and annotations.

Implement the gradient image and the original image Respectively with image segmentation. The gradient of the image segmentation's effect is better, so use the type to convert the gradient image [1]:

$$
G R A D(f)=\frac{1}{n} \sum_{i=1}^{n}\left(\left(f \oplus B_{i}\right)-\left(f \Theta B_{i}\right)\right) \Theta B_{i-1}
$$

GRAD shows morphological gradient, Bi structural elements can be any shape, but need to satisfy the relationship: $B_{0} \subseteq B_{1} \subseteq \cdots \subseteq B_{n}$.

\subsection{Excessive Segmentation Problem.}

To the small edge of the watershed algorithm has a better response, but the watershed algorithm's shortcomings are as follows:

(1)Sensitive to noise. In image, the existence of noise can affect image's gradient. It will cause deviation when split the contour.

(2)Are prone to excessive segmentation. Because there is noise, the influence of the texture details. There will be many false local minimum value, there may be a lot of excessive segmentation in the segmentation region.

(3)Low contrast image will lose important contour. Under low contrast, the region boundary pixels gradient value is low, Some important outline may be lost because of the gradient is not high [2].

Solve the problem of watershed algorithm caused by excessive segmentation, general methods are mainly as follows:

(1)The image preprocessing. Before implement the watershed algorithm segmentation, we should remove noise, morphological reconstruction, tag background operations such as outlook. Doing like that can reduce reception basin and then reduce the number of excessive region segmentation.

(2) Adding constraints. At the time of the partitioning, add constraints can restrict the segmentation process.

(3) Merge and process the image before image segmentation. If there are a lot of small areas during the initial segmentation, merging process will have a lot of calculation, merging rules is different and have different segmentation results [3].

This approach which was used is to modify gradient function and the specific approach is to process thresholding gradient image, in order to avoid gray tiny changes caused by the excessive segmentation. That is:

$$
g(x, y)=\max (\operatorname{grad}(f(x, y)), g(\theta))
$$

$\mathrm{g}(\theta)$ is the threshold.

In the process of thresholding gradient image, the selection of threshold will greatly influence the final effect of image segmentation. So the selection of appropriate threshold is important for the result of image segmentation. If the selection of threshold is too big, the wanted target's split may contain tiny edge, gray level difference has changed not big, selection of threshold value is too big though can reduce the influence of noise, and also will ignore these small edge, the edge will not be separated. If 
selecting threshold value is too small, can't effectively reduce the influence of noise, and can't completely solve the problem of excessive segmentation.

\section{The Hardware and Software Platform}

\subsection{Raspberry Pi.}

Raspberry $\mathrm{Pi}$ is a kind of computer card based on Linux operating system. It is designed in order to promote the development of computer science. Open source Linux operating system, colorful interface design and low price. More and more get the favor of students. And because of its small volume, the plenty of function advantages. Commonly used in intelligent household and robot development such control areas [4].

The robot in this paper, its hardware platform was built based on Raspberry Pi 2B, Raspberry Pi 2B the single-core processor performance has improved than before, speed up to six times more than before, it uses the quad-core processor Cortex-A7, 1 GB of RAM memory. An external Micro USB socket supplied $5 \mathrm{~V}$ power, a 4 USB sockets, a HDMI video socket, a RJ45 network card interface, a Micro SD card socket, a $3.5 \mathrm{~mm}$ audio output device etc. such external device. Raspberry Pi $2 \mathrm{~B}$ in addition to the performance has improved greatly, and has made great improvement on the software compatibility. In addition to the original Linux operating system, it is also compatible with the Android operating system and Windows operating system. Greatly expand the application between the crowd and applications.

We install Raspberry Pi official recommended operating system, Raspbian system. And use the wireless network card, USB camera. Under this system, study based on the $\mathrm{C}$ language as a development language and combined with OpenCV. VNC server can be used to realize the Raspberry Pi transmit files with Windows PC. Using remote login software PUTTY to login Raspberry Pi, and then develop and debug in the PC. And through the raspberries pi GPIO port control MCU development board, realize the robot's motion control in water Micro controller and steering engine control [5].

\subsection{Introduction of OpenCV.}

OpenCV's full name is open source computer vision library, was established in 1999 by Intel research center. OpenCV is a cross-platform open source computer vision library, you can run on Linux, Windows, Android operating system, composed of a series of $\mathrm{C}$ function and a small amount of $\mathrm{C}++$ class. OpenCV provides Python, Ruby, MATLAB language interface, it contains the function has more than 500, covering the many applications of computer vision, implements many general algorithm of image processing and computer vision [6].

\subsection{Microcontroller and Steering Gear.}

The movement of the robot is controlled by microcontroller steering gear rotation. Mainly made up of three steering gear. Robots are two steering gear respectively on both sides, and tail have a steering gear. On both sides of the steering gear mainly control rise and descend of the robot, and its tail steering gear controls the robot's forward and speed.

Mainly consists of steering wheel is reduction gear group, the position feedback potentiometer, dc motor, control circuit boards, this a few parts, etc. Working principle is through input control signals, control circuit, which can control electrical machine, electrical machine will drive a series of reduction gear group. And then output to the steering wheel.

Use PWM signal as the steering gear control signal, utilize the change of the duty ratio and change the position of the steering gear. PWM signal is periodic width adjustable square wave pulse signal, and makes the cycle of the PWM signal is $20 \mathrm{~ms}$, scope of the change of the pulse width is $0.5 \mathrm{~ms}-2.5$ ms, the steering gear output shaft Angle of 0-180 degrees. When the square wave pulse width is changed, changed the Angle of the steering gear shaft, is directly proportional to the Angle change with the change of the pulse width. Set an example for steering gear, the relationship between the output angle and the pulse width of input signal was shown in figure 2 . 


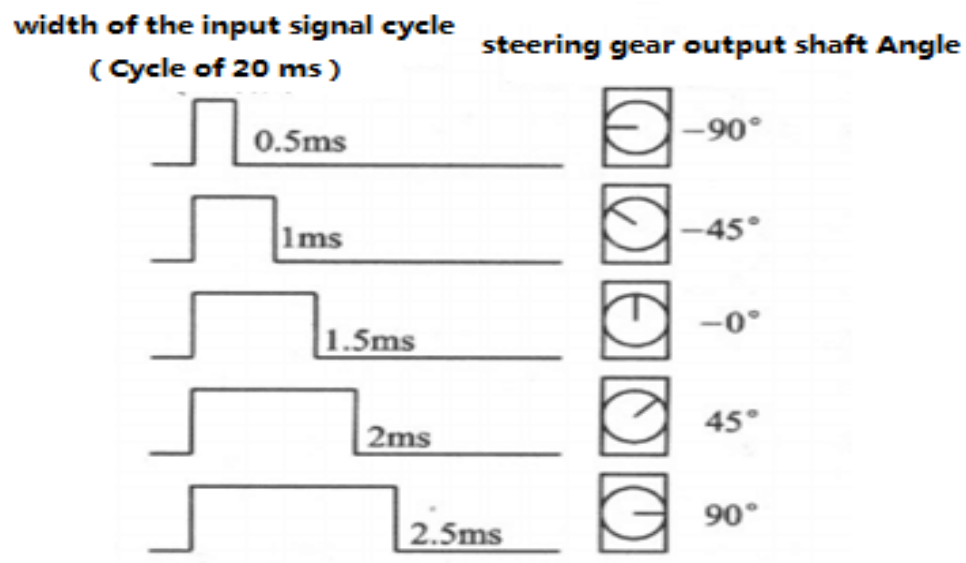

Fig. 2 steering Angle relationship between the input signal pulse widths

If provides fixed pulse width for steering gear, steering gear's output shaft will remain on a corresponding fixed angle. Until input a different width of the pulse signal, it will change the output angle and remains unchanged when change to a new corresponding position. Steering gear internal reference circuit and comparator: internal reference circuit can produce cycle of $20 \mathrm{~ms}$, width of 1.5 ms reference signal, the comparator will be compare input signal with reference signal. So as to determine the direction and size, then can produce motor rotation signal. So, steering gear is a kind of position servo system, It's maximum range of turn is 180 degrees, which can be used for robot's joints.

\section{The Experimental Test}

Test in the process of using the embedded platform is Raspberry Pi $2 \mathrm{~B}$. Raspberry Pi $2 \mathrm{~B}$ is a new generation of four nuclear Cortex-A7 processor with a 1GB of RAM. The operating system kernel use new ARM Cortex-A7 technology, with plenty of peripheral interface. Operating system is its official launch Raspbian system, using $\mathrm{C}$ language and $\mathrm{C}++$ programming language. Camera maximum resolution is $1280 \times 720$ and pixels is 5 million. The images taken by camera using watershed algorithm for image segmentation. Experimental testing is used indoor test pool, 3 meters long and 2 meters wide, depth of 0.35 meters. The result is shown in figure 3 , figure 4 .

Can effectively simulate the outdoor water environment. Raspberry Pi $2 \mathrm{~B}$ use watershed algorithm for image segmentation, through the Putty Vncserver remote SSH login, and the wireless network card image can be back to the PC. Can observe real-time, microcontroller control steering motion control the experimental effect is good at the same time.

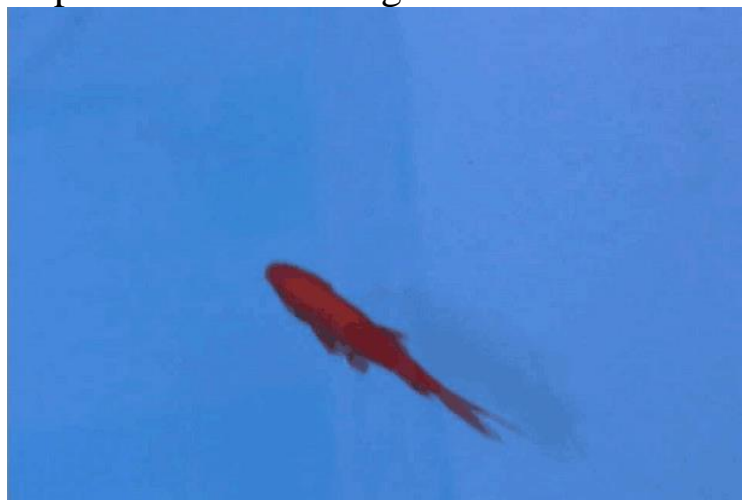

Fig. 3 The original image

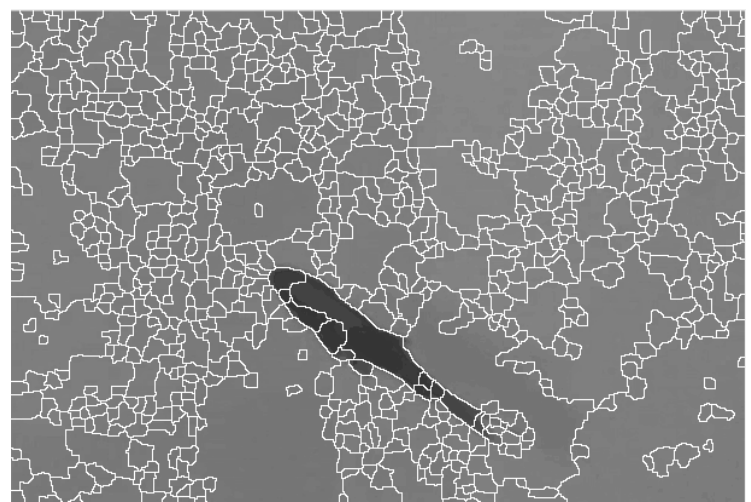

Fig. 4 The processed image

\section{Conclusion}

This paper introduces the Watershed algorithm, hardware platform and software platform of the robot. At last be tested through experiments. Under the simulation environment, the test result is good, can reach the expected goal. The robot can use its own cameras to transmit image to the notebook 
computer at real-time. Under Linux systems use OpenCV to watershed algorithm of image segmentation. You can see animals in the photos after processing. We also found some problems in the experimental process. If the pollutants in the water is a lot, after the watershed algorithm for image segmentation, pollutants may block the tested animals. This will lead to researchers make wrong judgment. Can also control the movement of the robot, from different perspectives to collect images to reduce the happening of this kind of situation. Robot can greatly reduce the workload and improve work efficiency, still need continuous improvement and innovation later.

\section{Acknowledgements}

This work was partially supported by National Nature Science Foundation (61673016), SWUN Construction Projects for Graduate Degree Programs (2017XWD-S0805), Advance Research Pro-gram of Electronic Science and Technology National Program (2017YYGZS16) and Sichuan Youth Science and technology innovation research team (2017TD0028), also was financially supported by students innovation training project of SWUN (201610656036).

\section{References}

[1]. Huijie Sun, Tingquan Deng, Yanchao Li. Improved watershed image segmentation algorithm. Vol. 25(2014), p. 857-864.

[2]. Zhihua Diao, Chunjiang Zhao, Xinyu Guo, et al. Study on the method of watershed algorithm is improved. Vol. 36(2010), p. 4-6.

[3]. Zheng Zhang, Hongxia Ni, Chunmiao Wan. Proficient in matlab digital image processing and recognition. Publishing House of People's posts And Telecommunications, Beijing, 2013.

[4]. Linwei Hao, Ying Liang. Based on Raspberry Pi+cloud service network monitoring and control system of furniture research and practical application. The Internet of things technology,2016.

[5]. Peter Membrey. Raspberry Pi study guide: based on Linux. Publishing House of People's posts And Telecommunications, Beijing, 2014.

[6]. Gary Bradski, Adrian Kaebler. Learing OpenCV. Publishing House of Tsinghua University, Beijing, 2009. 